Research Paper

\title{
H. pylori is related to NAFLD but only in female: A Cross-sectional Study
}

\author{
Jingwei Wang, Fengxiao Dong, Hui Su, Licun Zhu, Sujun Shao, Jing Wu, Hong Liu ${ }^{\bowtie}$ \\ Beijing Shijitan Hospital, Capital Medical University, Beijing, China. \\ $\triangle$ Corresponding author: Hong Liu: M.D., Chief physician, Professor, Department of Gastroenterology, Beijing Shijitan Hospital, Capital Medical University \\ Beijing, Beijing 100038, China. E-mail: liuhong_sjt@ccmu.edu.cn; Tel.: +86 13910285075; Fax: +86 1063926372. \\ (C) The author(s). This is an open access article distributed under the terms of the Creative Commons Attribution License (https://creativecommons.org/licenses/by/4.0/). \\ See http://ivyspring.com/terms for full terms and conditions.
}

Received: 2020.07.27; Accepted: 2021.03.01; Published: 2021.04.02

\begin{abstract}
Background: Recently, an increasing number of studies have focused on the extragastrointestinal effects of Helicobacter pylori $(\mathrm{H}$. pylori), including metabolic syndrome, fatty liver, and rheumatic and skin diseases. Nonalcoholic fatty liver disease (NAFLD) is a common chronic disease worldwide that conveys a heavy economic burden on patients and society. The aim of this study was to investigate the relationship between $\mathrm{H}$. pylori and NAFLD and to identify potential influencing factors.

Methods: We conducted a cross-sectional study of individuals who had undergone regular physical examinations at the Beijing Shijitan Hospital Health Examination Center from July to October 2018. We evaluated the associations between NAFLD and NAFLD with $\mathrm{H}$. pylori infection and related serum markers using multiple linear regression and logistic regression.

Results: There were significant relationships between $\mathrm{H}$. pylori infection status and NAFLD in females $(P=0.034)$ but not in males $(P=0.795)$ according to Fisher's exact test. The association persisted after further adjustment for metabolic variables, gastrin factors, and liver enzymes. Waist-to-Hip Ratio, Body Mass Index, triglycerides, High-density lipoprotein cholesterol, glucose, uric acid, alkaline phosphatase, and Alanine aminotransferase are related to NAFLD after adjusting for age or interaction between biochemical indexes.
\end{abstract}

Conclusion: $\mathrm{H}$. pylori infection is related to NAFLD in female patients. The relationship between $\mathrm{H}$. pylori and NAFLD may be mediated by markers of lipid metabolism and glycometabolism.

Key words: Female; H. pylori infection; Nonalcoholic fatty liver disease

\section{Introduction}

Helicobacter pylori (H. pylori), a Gram-negative, spiral-shaped microaerophilic bacterium, has been proven to be an important pathogen in gastrointestinal diseases [1]. Approximately $50 \%$ of the world's population has been affected by H. pylori infection, and the number of infected people was 4.4 billion in 2015. H. pylori infection rates vary across different regions, being $50.8 \%$ in developing countries and $34.7 \%$ in developed countries [2], and approximately 800 million Chinese people are affected by this disease. It may cause chronic inflammation of the gastric mucosa, which may lead to chronic atrophic gastritis, peptic ulcer diseases, and gastric cancer [3, 4]. Furthermore, the latest studies have identified associations between $\mathrm{H}$. pylori and extragastrointestinal effects, including metabolic syndrome [5], fatty liver [6], rheumatic and skin diseases [7] and so on. These parenteral diseases associated with $\mathrm{H}$. pylori infection seriously affect patients' general condition and convey a series of complications.

Nonalcoholic fatty liver disease (NAFLD) has been described as a condition of fatty infiltration of the liver in the absence of the other common causes of steatosis, for example, due to high alcohol consumption. The prevalence of NAFLD ranges from $6.3 \%$ to $33.0 \%$ worldwide [8]. Moreover, the prevalence of NAFLD in Asian countries has increased rapidly with a trend toward younger patients during the last two decades. Epidemiological 
studies show that NAFLD prevalence in the populations of Chengdu (southwest China), Shanghai (east China), Guangdong (south China) and central China is $12.5 \%, 15.0 \%, 17.0 \%$ and $24.5 \%$, respectively [9]. NAFLD includes a series of histological abnormalities, ranging from simple steatosis with traditionally benign clinical process to nonalcoholic steatohepatitis (NASH) characterized by inflammation, fibrosis and occasional cirrhosis. NASH may develop into decompensated liver disease and lead to liver failure, causing heavy economic burdens on both patients and society [9, 10]. Therefore, early detection and diagnosis of NAFLD is very important, and it is essential to identify additional risk factors for NFALD morbidity and progression.

Recognized risk factors for NAFLD include insulin resistance, obesity, hypertension, dyslipidemia, type 2 diabetes mellitus and metabolic syndrome [10]. Unfortunately, to date, the complex mechanism of NAFLD remains unclear. Recent studies have concentrated their attention on $\mathrm{H}$. pylori and NAFLD [10-12]. However, the relationship between these two entities remains controversial. A large-scale cohort study of 17,028 adults was conducted in Korea and verified that $H$. pylori infection was significantly associated with the development of NAFLD [11]. However, another study of 2051 participants concluded that Helicobacter pylori infection did not appear to increase the prevalence of NAFLD [12]. Given these discrepancies, more evidence is needed to verify the relationship between these entities. Previous studies have shown that $\mathrm{H}$. pylori infection contributes to development of NAFLD by altering lipid metabolism [11], promoting gastric atrophy [13] and so on. In this study, we performed a cross-sectional investigation to determine the relationship between $\mathrm{H}$. pylori and NAFLD and to identify potential influencing factors.

\section{Methods}

\section{Study population}

Briefly, men and women, aged 20 years old or older, were recruited who regularly had examinations at Beijing Shijitan Hospital Health Examination Center from July to October in 2018. Since we aimed to evaluate the relationship between $H$. pylori infection and NAFLD, we included a study population who underwent abdominal ultrasonography to assess the degree of steatosis in the liver and $\mathrm{H}$. pylori infection status $(\mathrm{n}=2896)$. We excluded patients with (1) alcohol intake ( $140 \mathrm{~g} /$ week for male and $70 \mathrm{~g} /$ week for female, $\mathrm{n}=629)$; (2) self-reported history of chronic liver disease or cirrhosis ( $\mathrm{n}=8)$; (3) self-reported history of cancer $(n=3)$; (4) positive diagnosis for hepatitis B or C virus $(n=3) ; \quad(4) \quad$ drug intake (drugs included antihypertension drugs, antidiabetic drugs, lipidlowering drugs, corticosteroids, and proton pump inhibitors $(n=287)$; (5) females who were pregnant or lactating $(\mathrm{n}=1) ;(6)$ a history of gastrointestinal surgery or trauma $(\mathrm{n}=0)$; and $(7)$ drug intake against H. pylori within the previous 1 month $(n=1)$. Furthermore, we excluded 66 participants with missing data. Finally, 1898 patients agreed to be in the study, and 66 people refused. A MOOSE flow chart is shown in Figure 1. This study was approved by the Ethics Committee of Beijing Shijitan Hospital Affiliated to Capital Medical University and was performed in accordance with the Declaration of Helsinki. Written informed consent was obtained from all participants.

\section{Data collection}

Questionnaires were completed by all study participants, including demographics (age, sex, race), smoking status, alcohol consumption, medicine use and personal medical history (obesity, hypertension, dyslipidemia, type 2 diabetes mellitus, metabolic syndrome, cancer). All blood measurements were performed with fresh serum obtained after a 12-hour fast to minimize the confounding effects of diurnal variation on hormone concentrations and included glucometabolic markers, liver function, renal function, lipid metabolism, ions (calcium, iron), tumor markers, pepsinogen (PG), and pro-gastrin-releasing peptide (proGRP). Anthropometric measurements, including waist circumference $(\mathrm{cm})$, blood pressure $(\mathrm{mmHg})$, body weight $(\mathrm{kg})$ and height $(\mathrm{cm})$, were measured by trained nurses using a standardized protocol. In addition, diastolic and systolic blood pressure were measured in the morning. Body mass index (BMI) was calculated by taking a person's weight, kilograms, divided by their height, in meters squared. Waist-to-hip ratio (WHR) was measured as waist circumference divided by hip circumference.

$\mathrm{H}$. pylori infection status was measure by ${ }^{13} \mathrm{C}$ breathing test on the same day in the morning when subjects had an empty stomach. The procedure was performed as follows: (1) two collecting bags were labeled with patient ID; (2) the air exhaled by the patient when they are breathing quietly was collected in one bag as the 0-minute sample; (3) the patient was given a urea $\left({ }^{13} \mathrm{C}\right)$ capsule with $50 \mathrm{ml}$ water, and 30 min later, the quiet breathing air was collected in the second bag (HCBT-01 tester; Shenzhen Zhonghe Headway Biological Technology Co. Ltd., China). The result is shown as delta over baseline (DOB), which indicates the ratio of ${ }^{13} \mathrm{CO} 2 /{ }^{12} \mathrm{CO} 2$ with the metabolic 
activity induced by labeled urea. A DOB greater than $4 \%$ is considered an active infection.

Diffuse fatty liver can be defined by the presence of at least two of three abnormal findings on abdominal ultrasonography: diffusely increased liver near field ultrasound echo ('bright liver'), liver echo greater than kidney; vascular blurring and the gradual attenuation of far field ultrasound echo [14]. NAFLD patients were confirmed by the following criteria: (1) diffuse fatty liver in ultrasound; (2) no significant alcohol consumption ( $<140 \mathrm{~g}$ (male) or $<70$ $\mathrm{g}$ (female) alcohol consumption per week); and (3) no coexisting causes of chronic liver disease, such as hepatitis C, medication, parenteral nutrition, Wilson's disease or severe malnutrition [15]. Participants with fatty liver were divided into mild, moderate and severe categories according to the ultrasonic findings. Steatosis is graded as follows: Absent (score 0) when the echotexture of the liver is normal; mild (score 1), when there is a slight and diffuse increase of liver echogenicity with normal visualization of the diaphragm and of the portal vein wall; moderate (score 2), in case of a moderate increase of liver echogenicity with slightly impaired appearance of the portal vein wall and the diaphragm; severe (score 3), in case of marked increase of liver echogenicity with poor or no visualization of portal vein wall, diaphragm, and posterior part of the right liver lobe [16]. All ultrasonography was performed by two professional ultrasound doctors who were uniformly trained to diagnose fatty liver.

The study population was further divided into the risk and nonrisk groups. The risk group was defined as the simultaneous occurrence of one or more of the following risk factors: (1) FPG $\geq 6.10$ $\mathrm{mmol} / \mathrm{L}$; (2) $\mathrm{TG} \geq 1.70 \mathrm{mmol} / \mathrm{L}$; (3) $\mathrm{TC} \geq 5.20$ mmol/L; (4) HDL-C < $1.00 \mathrm{mmol} / \mathrm{L}$; (5) LDL-C $\geq 3.10$ $\mathrm{mmol} / \mathrm{L} ; \quad$ (6) $\quad$ BMI $\geq 24.90 \mathrm{~kg} / \mathrm{m}^{2}$; (7) waist

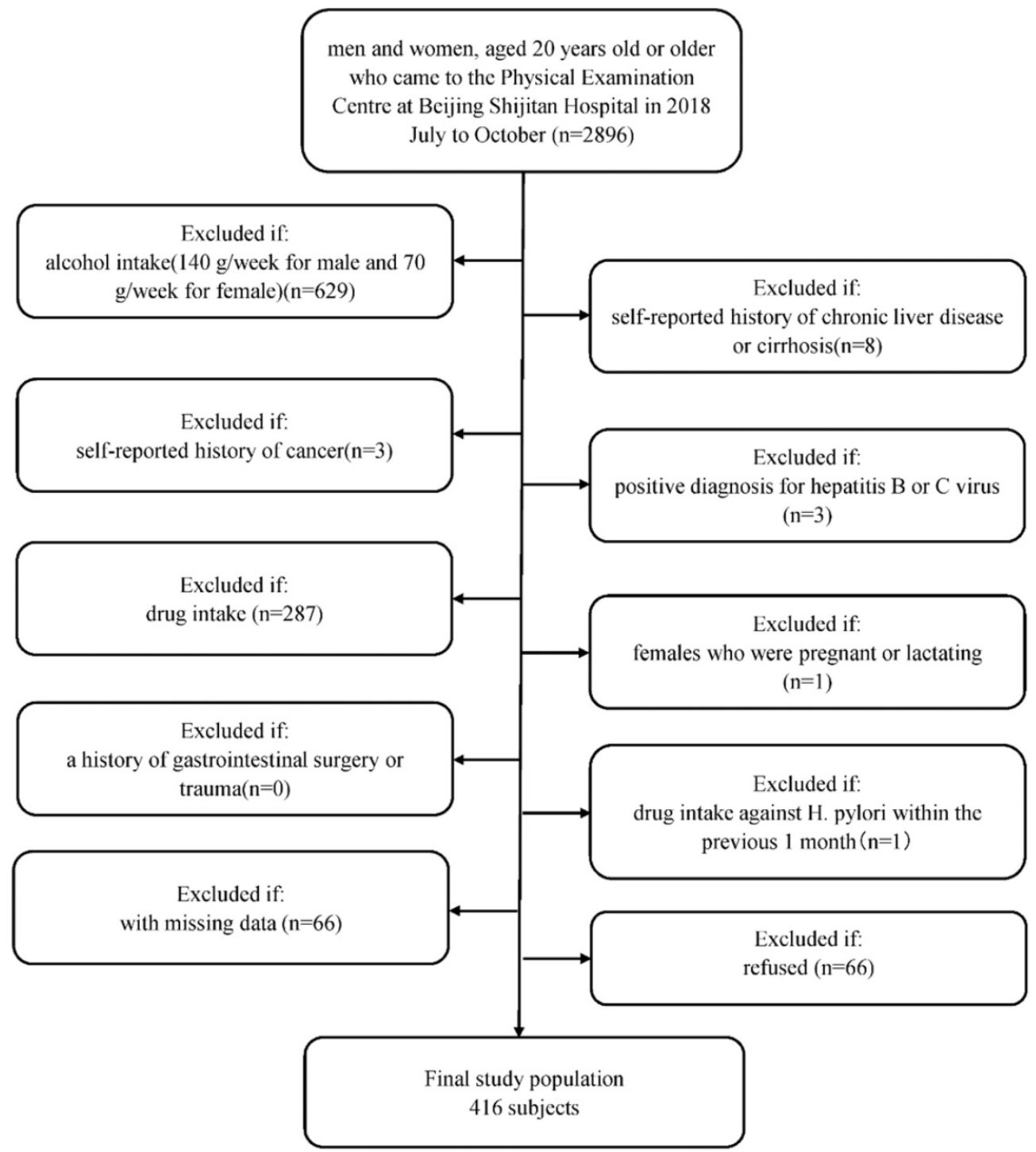

Figure 1. Flow diagram of study participants. Flow chart of selection of the included subjects. 
circumference $\geq 90 \mathrm{~cm}$, triglyceride levels $\geq 150$ $\mathrm{mg} / \mathrm{dL}, \mathrm{HDL}$ cholesterol $\leq 40 \mathrm{mg} / \mathrm{dL}$, systolic blood pressure $\geq 130 \mathrm{mmHg}$, diastolic blood pressure $\geq 85$ $\mathrm{mmHg}$, FBG levels $\geq 100 \mathrm{mg} / \mathrm{dL}$ (satisfied more than three). Those who did not satisfy the risk criteria were allocated into the nonrisk group [11].

\section{Statistical analyses}

We used SPSS statistical software version 22.0 for data analyses. Continuous variables are reported as the means \pm standard deviation, whereas categorical variables are presented as percentages. The Kolmogorov-Smirnov test was used to verify whether the data was normally distributed, and all continuous variables that did not conform to a normal distribution underwent transformation for analysis. Study subjects were classified into $\mathrm{H}$. pylori positive and $\mathrm{H}$. pylori negative according to $\mathrm{H}$. pylori infection status, and summary and grouping data for baseline characteristics (the laboratory examination) were compared using t-test for continuous variables and Fisher's exact test for categorical variable, which is shown in Table 1.

Multivariate-adjusted odds ratios (ORs) and 95\% confidence intervals (CIs) were calculated using logistic regression in the mild, moderate and severe fatty liver groups. A logistic regression model adjusted for age or interaction between biochemical indexes was used to assess associations between NAFLD and $H$. pylori infection status by dichotomizing factor levels and calculating the odds ratios (ORs).

We fit additional models adjusted for metabolic factors, gastrin factors and liver function factors that could be potential confounders or mediators of the association between $\mathrm{H}$. pylori infection and incident NAFLD. Model 1 was adjusted for confounding factors and fasting blood glucose, Ghb, TC, TG, LDL-C, HDL-C, and UA; model 2 was further adjusted for BMI, WHR, SBP, and DBP; model 3 was further adjusted for AST, ALT, and GGT; Finally, model 4 was adjusted for model 3 plus PGI, PGII, PGI/PGII, and proPG.

To further analyze the relationship between $\mathrm{H}$. pylori infection and NAFLD, we analyzed the relationship between the risk and nonrisk group populations based on the degree of NAFLD. All models excluded age as a confounder. P-value $<0.05$ was considered statistically significant.

\section{Results}

Data were analyzed from 1898 patients, age 22 to 67 years old, who were examined at Beijing Shijitan Hospital Health Examination Center. The baseline characteristics of the participants are shown in Table
1. The mean participant age was $37.19 \pm 0.170,1217$ $(64.1 \%)$ were male, and $681(35.9 \%)$ were female. Among the study population, 25 (1.3\%) participants had severe NAFLD, 174 (9.2\%) had moderate NAFLD, 306 (16.1\%) had mild NAFLD and 1393 (73.4\%) did not have NAFLD. The mean age of the NAFLD and non-NAFLD groups was $36.42 \pm 7.031$ and $39.31 \pm 8.010$, and this difference was statistically significant $(\mathrm{t}=-7.154, \quad P<0.001)$. There was also a significant difference between male and the female participants $(P<0.001)$, which is shown in Table 2.

Table 1. Baseline characteristics of the patients according to the H. pylori infection statue

\begin{tabular}{|c|c|c|c|c|}
\hline & Total & H. pylori + & H. pylori - & $P$-value \\
\hline Age & $37.19 \pm 0.170$ & $37.04 \pm 0.289$ & $37.28 \pm 0.210$ & 0.452 \\
\hline \multicolumn{5}{|l|}{ Sex } \\
\hline Female & $35.9 \%$ & $33.5 \%$ & $37.2 \%$ & 0.107 \\
\hline Male & $64.1 \%$ & $66.5 \%$ & $62.8 \%$ & \\
\hline SP & $114.26 \pm 0.330$ & $114.68 \pm 0.574$ & $114.02 \pm 0.401$ & 0.476 \\
\hline BP & $68.400 \pm 0.244$ & $68.580 \pm 0.417$ & $68.300 \pm 0.301$ & 0.894 \\
\hline BMI & $23.165 \pm 0.078$ & $23.229 \pm 0.126$ & $23.114 \pm 0.099$ & 0.300 \\
\hline WHR & $0.873 \pm 0.001$ & $0.873 \pm 0.002$ & $0.873 \pm 0.002$ & 0.533 \\
\hline ALT & $22.861 \pm 0.207$ & $23.087 \pm 0.606$ & $22.732 \pm 0.470$ & 0.260 \\
\hline AST & $19.856 \pm 0.207$ & $20.255 \pm 0.355$ & $19.628 \pm 0.255$ & 0.156 \\
\hline UA & $360.730 \pm 2.099$ & $366.520 \pm 3.427$ & $357.440 \pm 2.650$ & 0.019 \\
\hline $\mathrm{TC}$ & $4.562 \pm 0.019$ & $4.559 \pm 0.032$ & $4.563 \pm 0.023$ & 0.836 \\
\hline TG & $1.345 \pm 0.022$ & $1.410 \pm 0.044$ & $1.308 \pm 0.025$ & 0.210 \\
\hline HDL-C & $1.306 \pm 0.007$ & $1.285 \pm 0.010$ & $1.318 \pm 0.008$ & 0.024 \\
\hline LDL-C & $2.408 \pm 0.013$ & $2.409 \pm 0.227$ & $2.408 \pm 0.017$ & 0.985 \\
\hline GLU & $4.714 \pm 0.020$ & $4.760 \pm 0.038$ & $4.687 \pm 0.223$ & 0.348 \\
\hline ALP & $59.660 \pm 0.454$ & $60.020 \pm 0.630$ & $59.450 \pm 0.615$ & 0.188 \\
\hline GGT & $24.620 \pm 0.607$ & $26.060 \pm 1.350$ & $23.800 \pm 0.561$ & 0.397 \\
\hline PGI (ng/ml) & $48.630 \pm 24.077$ & $55.317 \pm 20.816$ & $44.819 \pm 24.971$ & 0.000 \\
\hline PG2 (ng/ml) & $8.361 \pm 6.275$ & $11.051 \pm 6.151$ & $6.827 \pm 5.815$ & 0.000 \\
\hline PG1/PG2 & $6.437 \pm 1.800$ & $5.633 \pm 1.810$ & $6.895 \pm 1.626$ & 0.000 \\
\hline proGRP $(\mathrm{pg} / \mathrm{mL})$ & $28.080 \pm 0.210$ & $28.194 \pm 0.375$ & $28.015 \pm 0.252$ & 0.952 \\
\hline Ghb (\%) & $5.379 \pm 0.013$ & $5.406 \pm 0.025$ & $5.364 \pm 0.134$ & 0.676 \\
\hline
\end{tabular}

Abbreviations as in Table 1 and Table 2; OR, odds ratio;

Bold indicates statistically significant values.

BMI, Body Mass Index; WHR, waist-to-hip ratio; TC, total cholesterol; Ghb, glycosylated hemoglobin; TG, triglyceride; UA, uric acid; AST, Aspartate aminotransferase; ALT, Alanine aminotransferase; Ca, calcium; DP, diastolic blood pressure; SP, Systolic pressure; $\mathrm{Hb}$, hemoglobin; HDL-C, High-density lipoprotein cholesterol; LDL-C, Low-density lipoprotein cholesterol; UA, Uric acid; GLU, glucose; PG, pepsinogen; proGRP, Pro-gastrin-releasing Peptide; ALP, alkaline phosphatase;GGT, $\gamma$-glutamyl transpeptidase.

Table 2. The relationship between the $H$. pylori infection and the NAFLD level in different genders

\begin{tabular}{llll}
\hline & H. pylori infection $(-)$ & H. pylori infection $(+)$ & $P$-value \\
\hline Female & & & \\
Normal & $418(92.9 \%)$ & $202(87.4 \%)$ & 0.034 \\
mild NAFLD & $19(4.2 \%)$ & $13(5.6 \%)$ & \\
moderate NAFLD & $13(2.9 \%)$ & $15(6.5 \%)$ & \\
severe NAFLD & $0(0.0 \%)$ & $1(0.4 \%)$ & 0.795 \\
Male & & & \\
Normal & $485(63.9 \%)$ & $288(62.9 \%)$ & \\
mild NAFLD & $168(22.1 \%)$ & $106(23.1 \%)$ & \\
moderate NAFLD & $93(12.3 \%)$ & $53(11.6 \%)$ & \\
severe NAFLD & $13(1.7 \%)$ & $11(2.4 \%)$ & \\
\hline
\end{tabular}

Bold indicates statistically significant values. 
Among all patients, 689 participants presented with $\mathrm{H}$. pylori infection, and 1209 participants did not have an $\mathrm{H}$. pylori infection. The mean age of patients with and without $H$. pylori infection was $37.04 \pm 0.289$ (66.5\% male), $37.28 \pm 0.210$ (62.8\% male). Table 1 shows that UA $(\mathrm{t}=-2.082, P=0.038)$ and HDL-C $(\mathrm{t}=-2.449$, $P=0.014)$ were significantly different between those with $\mathrm{H}$. pylori infection and those without $\mathrm{H}$. pylori infection, but there was no significant difference between $\mathrm{H}$. pylori infection status and NAFLD.

In Table 2, by separating the study population according to sex, we found that there was a significant relationship between $\mathrm{H}$. pylori infection status and NAFLD in female patients $(P=0.034)$ but not in male patients $(P=0.795)$.
As shown in Table 3, in a separate logistic regression, there was a significant positive association of WHR (OR=0.041, $P<0.001)$, BMI (OR=0.032, $P$ $<0.001)$, TG (OR=0.087, $P<0.001)$, HDL-C $(\mathrm{OR}=0.086$, $P<0.001)$, GLU (OR=0.086, $P<0.001)$, UA (OR=0.138, $<0.001)$, ALP $(\mathrm{OR}=0.124, P=0.004)$, ALT $(\mathrm{OR}=0.124, P$ $=0.004)$ and $\mathrm{C} 13(\mathrm{OR}=0.473, P=0.007)$ with NAFLD after adjusting for age in female patients. Furthermore, WHR (OR=0.097, $P<0.001)$, BMI $(\mathrm{OR}=0.074, \quad P<0.001)$, TG $(\mathrm{OR}=0.165, \quad P<0.001)$, HDL-C (OR=0.309, $P<0.001)$, GLU (OR=0.197, $P$ $=0.042)$, UA $(\mathrm{OR}=0.240, P<0.001)$ and ALP $(\mathrm{OR}=0.144$, $P=0.008)$, and ALT (OR=0.076, $P=0.001)$ were significantly associated in the biochemical indexes model in female patients with NAFLD.

Table 3. The relationship between NAFLD statue and the potential biomarker

\begin{tabular}{|c|c|c|c|c|c|}
\hline \multirow[t]{2}{*}{ Characteristics } & Model 1† & Model $2 \ddagger$ & \multirow[t]{2}{*}{ Characteristics } & Model 1† & Model $2 \ddagger$ \\
\hline & OR $(95 \% \mathrm{CI})$ & OR $(95 \% \mathrm{CI})$ & & OR $(95 \% \mathrm{CI})$ & OR $(95 \% \mathrm{CI})$ \\
\hline WHR & & & BMI $\left(\mathrm{kg} / \mathrm{m}^{2}\right)$ & & \\
\hline Q1 & $0.041(0.012,0.133)$ & $0.378(0.285,0.503)$ & Q1 & $0.032(0.016,0.064)$ & $0.201(0.151,0.268)$ \\
\hline Q2 & 0 & 0 & Q2 & 0 & 0 \\
\hline$P$-value & 0.000 & 0.000 & $P$-value & 0.000 & 0.000 \\
\hline $\mathrm{SP}(\mathrm{mmHg})$ & & & $\mathrm{BP}(\mathrm{mmHg})$ & & \\
\hline Q1 & $0.284(0.047,1.726)$ & $0.719(0.352,1.467)$ & Q1 & $0.061(0.007,0.553)$ & $0.656(0.313,1.376)$ \\
\hline Q2 & 0 & 0 & Q2 & 0 & 0 \\
\hline$P$-value & 0.172 & 0.364 & $P$-value & 0.013 & 0.265 \\
\hline TC (mmol/L) & & & TG (mmol/L) & & \\
\hline Q1 & $0.359(0.176,0.733)$ & $1.758(0.607,5.099)$ & Q1 & $0.087(0.045,0.169)$ & $0.165(0.076,0.355)$ \\
\hline Q2 & 0 & 0 & Q2 & 0 & 0 \\
\hline$P$-value & 0.005 & 0.299 & $P$-value & 0.000 & 0.000 \\
\hline HDL-C (mmol/L) & & & LDL-C (mmol/L) & & \\
\hline Q1 & $5.830(2.959,11.484)$ & $0.309(0.110,0.868)$ & Q1 & $0.605(0.154,2.377)$ & $0.384(0.122,1.208)$ \\
\hline Q2 & 0 & 0 & Q2 & 0 & 0 \\
\hline$P$-value & 0.000 & 0.026 & $P$-value & 0.472 & 0.102 \\
\hline GLU (mmol/L) & & & Ghb (\%) & & \\
\hline Q1 & $0.086(0.023,0.321)$ & $0.197(0.041,0.942)$ & Q1 & $0.711(0.177,2.849)$ & $0.342(0.096,1.219)$ \\
\hline Q2 & 0 & 0 & Q2 & 0 & 0 \\
\hline$P$-value & 0.000 & 0.042 & $P$-value & 0.630 & 0.098 \\
\hline UA (umol/L) & & & $\operatorname{ALP}(U / L)$ & & \\
\hline Q1 & $0.138(0.075,0.252)$ & $0.240(0.120,0.479)$ & Q1 & $0.124(0.030,0.514)$ & $0.144(0.035,0.599)$ \\
\hline Q2 & 0 & 0 & Q2 & 0 & 0 \\
\hline$P$-value & 0.000 & 0.000 & $P$-value & 0.004 & 0.008 \\
\hline GGT (U/L) & & & ALT (U/L) & & \\
\hline Q1 & $0.409(0.078,2.158)$ & $2.497(0.294,21.221)$ & Q1 & $0.057(0.017,0.195)$ & $0.076(0.016,0.357)$ \\
\hline Q2 & 0 & 0 & Q2 & 0 & 0 \\
\hline$P$-value & 0.292 & 0.402 & $P$-value & 0.000 & 0.001 \\
\hline AST (U/L) & & & PGI (ng/ml) & & \\
\hline Q1 & $0.069(0.014,0.345)$ & $0.420(0.045,3.896)$ & Q1 & $1.857(1.064,3.245)$ & $1.597(0.786,3.245)$ \\
\hline Q2 & 0 & 0 & Q2 & 0 & 0 \\
\hline$P$-value & 0.001 & 0.445 & $P$-value & 0.029 & 0.196 \\
\hline PGII (ng/ml) & & & PGI/PGII & & \\
\hline Q1 & $1.252(0.721,2.175)$ & $0.962(0.432,2.140)$ & Q1 & $1.266(0.736,2.179)$ & $1.212(0.628,2.335)$ \\
\hline Q2 & 0 & 0 & Q2 & 0 & 0 \\
\hline$P$-value & 0.424 & 0.923 & $P$-value & 0.393 & 0.567 \\
\hline proPG $(\mathrm{pg} / \mathrm{ml})$ & & & $\mathrm{C} 13$ & & \\
\hline Q1 & $1.266(0.736,2.179)$ & $1.212(0.628,2.335)$ & Q1 & $0.473(0.274,0.817)$ & - \\
\hline Q2 & 0 & 0 & Q2 & 0 & \\
\hline$P$-value & 0.393 & 0.567 & $P$-value & 0.007 & \\
\hline
\end{tabular}

Abbreviations as in Table 1; OR, odds ratio;

The model $1 \dagger$ only used age as a covariable to the logistic regression models;

In the model $2 \ddagger$, the variables were divided into four groups: basic information (WHR, BMI, SP, BP); metabolic index (TC, TG, HDL-C, LDL-C, GLU, Ghb, UA), Liver related indicators (ALP, GGT, ALT) and gastrin related indexes (PGI, PGII, PGI/PGII, proPG). When analyzed one of the variables, other variables in the same group and age were included in the model as covariables;

Bold indicates statistically significant. 
Table 4. Mediation analysis of the association between H.pylori status and the development of nonalcoholic fatty liver disease (NAFLD)

\begin{tabular}{llll}
\hline & OR & $95 \%$ CI & $P$ \\
\hline Model 0, aHRa (95\% CI) & 0.473 & $0.274,0.817$ & 0.007 \\
Model 1, aHRa (95\% CI) & 0.517 & $0.274,0.974$ & 0.041 \\
Model 2, aHRa (95\% CI) & 0.395 & $0.164,0.949$ & 0.038 \\
Model 3, aHRa (95\% CI) & 0.395 & $0104,0.807$ & 0.018 \\
Model 4, aHRa (95\% CI) & 0.221 & $0.067,0.736$ & 0.014 \\
\hline
\end{tabular}

Model 0: adjusted for age;

Model 1: Model 0 plus adjustment for fasting blood glucose, Ghb, TC, TG, LDL-C, HDL-C, UA;

Model 2: Model 1 plus adjustment for BMI, WHR, SBP, and DBP;

Model 3: Model 2 plus adjustment for AST, ALT, and GGT;

Model 4: Model 3 plus adjustment for PGI, PGII, PGI/PGII, proPG.

To explore whether the increased incidence of NAFLD with $\mathrm{H}$. pylori infection was mediated by metabolic risk factors, gastrin factors or liver enzymes, we conducted additional analyses adjusted for potential mediators in female participants (Table 4). After adjusting for metabolic variables, such as fasting blood glucose, Ghb, TC, TG, LDL-C, HDL-C, and UA (Model 3), the association between $\mathrm{H}$. pylori infection and incident NAFLD was attenuated but remained statistically significant, suggesting mediation by these metabolic factors.

We next separated the study population into risk and nonrisk groups among female participants, which is presented in Table 5. In the risk group, 25 subjects were classified as having moderate NAFLD, 26 subjects as mild NAFLD and 146 as normal liver. In the nonrisk group, 1 subject had severe NAFLD, 3 subjects had moderate NAFLD, 6 subjects had mild NAFLD and 474 had normal liver. We analyzed the relationship between $\mathrm{H}$. pylori infection and the risk/ nonrisk groups, observing a significant difference between $\mathrm{H}$. pylori infection $(\mathrm{OR}=0.502,95 \% \mathrm{CI}$ 0.263-0.961 $P=0.037$ ) and the female participants with risk. There was a trend that did not reach significance between $\mathrm{H}$. pylori infection (OR=0.512, 95\% CI 0.083, $1.104 P=0.037$ ) and the nonrisk group in female participants. All analyses excluded age as a confounder.

\section{Discussion}

NAFLD is an important health and societal burden in Asian people, both females and males. There are numerous NAFLD-related risk factors, including insulin resistance, obesity, hypertension, dyslipidemia, type 2 diabetes mellitus and metabolic syndrome. Therefore, we conducted a cross-sectional study to identify risk factors for NAFLD.

In this study, we found that $\mathrm{H}$. pylori infection is a risk factor for NAFLD in female patients. After further adjustment of the model for metabolic risk factors, liver enzymes and pepsin related factors, this association persisted. Adjustment for metabolic variables, such as fasting blood glucose, Ghb, TC, TG, LDL-C, HDL-C, and UA, reduced the association between $\mathrm{H}$. pylori infection and NAFLD, but the association was still statistically significant, verifying our result. However, the relationship between $\mathrm{H}$. pylori and NAFLD remains controversial. A large-scale cohort study in Korea contends that $\mathrm{H}$. pylori infection is significantly associated with the development of NAFLD, independent of metabolic and inflammatory risk factors [11]. Another metaanalysis including 13 observations concluded that $\mathrm{H}$. pylori infection is associated with NAFLD in middleaged individuals [17]. However, another large-scale cross-sectional study in Japan reported that H. pylori infection was not associated with fatty liver disease [18]. In China, a cross-sectional study including 21456 participants reported that $\mathrm{H}$. pylori infection was not independently associated with the risk of NAFLD in apparently healthy subjects [19]. The heterogeneity of research results among different studies may be caused by different sample sizes, sample baseline characteristics, Mets diagnostic criteria, follow-up time differences and exclusion of different confounding factors.

Table 5. Definition of NAFLD risk group and the relationship between $\mathrm{H}$. pylori infection and NAFLD in risk and non-risk group

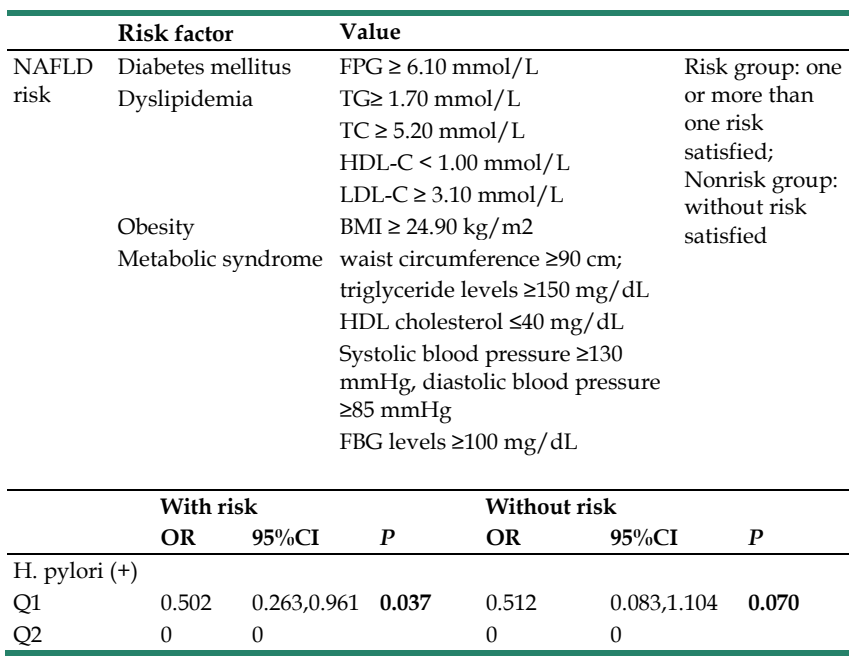

Abbreviations as in Table 1; OR, odds ratio;

Bold indicates statistically significant.

Our research found that $\mathrm{H}$. pylori are related to NAFLD only in female patients, which we believe is for the following reasons. First, due to the protection afforded by sex hormones in women, premenopausal women are less likely to suffer from NAFLD than men [20]. NAFLD guidelines also state that being male is a risk factor for NAFLD; therefore, we believe that there are differences between men and women with respect 
to the factors that influence NAFLD. Second, due to cultural factors, people tend to conceal their alcohol intake history. Although we repeatedly stressed the importance of the participants revealing the amount of alcohol they drank, we cannot exclude the possibility of concealment of daily alcohol intake by some participants, which may lead to biased results. Meanwhile, female participants usually drink less than men, so this effect will likely be smaller in women. Last but not least, essays support the idea that men are more likely to suffer from secondary diseases, for example, rheumatoid arthritis, alcoholism, excessive smoking, and gonadal deficiencies, among others [21], which may predispose to NAFLD. The mechanism of NAFLD is very complex and is not entirely clear at present. Our study demonstrated that NAFLD is related to $\mathrm{H}$. pylori in female, but not male, patients. We suspect this might be due to differences in the etiology of NAFLD and influencing factor between male and female patients. However, we did not find any literature to support this connection, which needs additional systematic research to confirm.

In male participants, we found that BMI, WHR, ALT, UA, TG, HDL-C, and Ghb were related to NAFLD. In female participants, we found that BMI, WHR, ALT, UA, GLU, ALP, and C13 were related to NAFLD. TG and HDL-C had a trend toward a significant difference in female participants, indicating that these metabolic factors may be intermediates in the relationship between $\mathrm{H}$. pylori and NAFLD. There is increasing evidence that NAFLD is associated with obesity, dyslipidemia, diabetes and insulin resistance and is considered to be a liver manifestation of metabolic syndrome [18], which is supported by our results.

After excluding age, sex, systolic blood pressure, TC, TG, LDL-C, HDL-C, ALT, AST, GGT as confounders, a significant association between $\mathrm{H}$. pylori and NAFLD persisted. Although the relationship between $\mathrm{H}$. pylori and NAFLD still needs further study, experts think the following reason may explain the connection. First, H. pylori infection is thought to increase the rate of metabolic syndrome $[22,23]$. Seon Hee Lim conducted a multicenter study in Korea and found that $\mathrm{H}$. pylori infection plays an independent role in the pathogenesis of metabolic syndrome. Furthermore, metabolic syndrome is thought to be related to NAFLD [24], which is supported by our results as well. In addition, $\mathrm{H}$. pylori infection may influence lipid metabolism, including low HDL-C, high LDL-C and TG [25-27], as well as abnormal glycometabolism, including increased fasting glucose and insulin levels [28]. Our research revealed significant differences in TG and
HDL-C in different degrees of fatty liver patients, and we got same result as the previous research [11]. Second, Helicobacter pylori infection may induce NAFLD through transmission of inflammatory stimuli (e.g., lipopolysaccharide (LPS)) directly into the portal venous circulation. $\mathrm{H}$. pylori infection has also been shown to exert systemic effects through the release of vasoactive substances and inflammatory cytokines (e.g., by increasing levels of interleukin-6, tumor necrosis factor-alpha and other proinflammatory cytokines), which may play an important role in the development of NAFLD by enhancing oxidative stress, promoting the transition to adipogenesis and increasing the chance of insulin resistance [6, 29-34]. Last but not least, gastric atrophy caused by Helicobacter pylori may lead to gastric acid loss, subsequent small intestinal bacterial overgrowth (SIBO), and increased intestinal permeability, which may increase portal endotoxins [13, 33, 35]. Cytotoxin associated gene $\mathrm{A}$ antigen ( $\mathrm{Cag} \mathrm{A})$, the virulence factor of $\mathrm{H}$. pylori, has been proven to alter the gut microbiota, resulting in exacerbation of cell proliferation and immune phenotypes [36]. Although $H$. pylori infection may have a beneficial effect on serum liver enzyme levels and other surrogate markers of NAFLD, the effect of early eradication therapy is still insufficient. The limited available research indicates that $\mathrm{H}$. pylori eradication therapy improves nonalcoholic fatty liver disease fibrosis score and may led to greater improvement in HOMA-IR [37, 38].

Despite these relevant findings, our study has several limitations. First, because most patients cannot remember when they were first diagnosed with $\mathrm{H}$. pylori infection, we were not able to obtain the time of $\mathrm{H}$. pylori infection, so different infection times may have had an impact on the results. Second, the sample size of our data was not large enough, and the study population only included participants from Beijing Shijitan Hospital, meaning that there might have been confounding factors due to differences in the distribution of hospital study populations. Third, we did not obtain a study population with successful eradication of $\mathrm{H}$. pylori infection. Additional largescale studies in the general population are needed to validate our results. Fourth, we only used ultrasound as a criterion for the diagnosis of fatty liver; however, the ultrasound results were completed by two professional ultrasound doctors, which we think were accurate. Fifth, we did not measure participant study indicators repeatedly. In addition, we identified some differences between men and women but failed to further explore them.

In conclusion, this study demonstrates that $\mathrm{H}$. pylori infection is related to NAFLD in female 
patients. This effect might be exerted through markers of lipid metabolism and glycometabolism. Considering the limited clinical data in this study, it is necessary to further investigate the relationship between $H$. pylori infection and NAFLD, which undoubtedly will provide new insight for effective prevention and treatment of NAFLD.

\section{Abbreviations}

Lumbar dual-energy X-ray absorptiometry (DXA), odds ratios (ORs), confidence intervals (CIs), Helicobacter pylori (H. pylori), selective estrogen receptor modulators (SERMs), pro-gastrin-releasing peptide (proGRP), Body Mass Index (BMI), Waist-to-Hip Ratio (WHR), bone mineral density (BMD), World Health Organization (WHO), standard deviation (SD), total cholesterol (TC), triglycerides (TG), uric acid (UA), Low-density lipoprotein cholesterol (LDL-C), glycosylated haemoglobin (Ghb), High-density lipoprotein cholesterol (HDL-C), glucose (GLU), pepsinogen (PG), calcium (CA), diastolic blood pressure (DP), Haemoglobin $(\mathrm{Hb})$, systolic blood pressure (SP), 13C breathing test positive (C13). Pepsinogen (PG); alkaline phosphatase (ALP); GGT, r-glutamyl transpeptidase.

\section{Acknowledgements}

\section{Funding}

The study was supported by a program from the Beijing City Health System "215" High Levels of Health Technical Personnel Training Aid and the Capital Clinical Characteristic Applied Research Project (No. Z181100001718120). Funds are used for the data collection portion.

\section{Ethics approval and consent to participate}

All the ethics approval has been given by the ethics committee of Beijing Shijitan hospital affiliated to capital medical university and have been performed in accordance with the Declaration of Helsinki. We used the participants data by anonymous. All involved study populations have given their informed written consent before included in the study.

\section{Availability of data and materials}

The datasets analyzed during the current study are not publicly available because it includes the study population personal information which is illegal to open but are available from the corresponding author on reasonable request.

\section{Authors' contributions}

$\mathrm{LH}$ and WJ made substantial contributions to the design of the work; SSJ has provided the data; ZLC has made contributions to the collection of data; DFX has analyzed and interpreted of the data; WJW has drafted the manuscript; $\mathrm{SH}$ has helped to revise the manuscript. All authors have read and approved the manuscript.

\section{Competing Interests}

The authors have declared that no competing interest exists.

\section{References}

1. Glaser DL, Kaplan FS. Osteoporosis. Definition and clinical presentation. Spine (Phila Pa 1976). 1997;22(24 Suppl):12s-6s

2. Zamani M, Ebrahimtabar F, Zamani V, et al. Systematic review with meta-analysis: the worldwide prevalence of Helicobacter pylori infection. Alimentary pharmacology \& therapeutics. 2018;47(7):868-76.

3. Malfertheiner P, Megraud F, O'Morain CA, et al. Management of Helicobacter pylori infection - the Maastricht IV/ Florence Consensus Report. Gut. 2012;61(5):646-64.

4. Matsuhisa T, Aftab H. Observation of gastric mucosa in Bangladesh, the country with the lowest incidence of gastric cancer, and Japan, the country with the highest incidence. Helicobacter. 2012;17(5):396-401.

5. Upala S, Jaruvongvanich V, Riangwiwat $\mathrm{T}$, et al. Association between Helicobacter pylori infection and metabolic syndrome: a systematic review and meta-analysis. Journal of digestive diseases. 2016;17(7):433-40.

6. Tang DM, Kumar S. The Association Between Helicobacter pylori Infection and Nonalcoholic Fatty Liver Disease. Current gastroenterology reports. 2017;19(2):5.

7. Smyk DS, Koutsoumpas AL, Mytilinaiou MG, et al. Helicobacter pylori and autoimmune disease: cause or bystander. World journal of gastroenterology. 2014;20(3):613-29.

8. Vernon G, Baranova A, Younossi ZM. Systematic review: the epidemiology and natural history of non-alcoholic fatty liver disease and non-alcoholic steatohepatitis in adults. Alimentary pharmacology \& therapeutics. 2011;34(3):274-85.

9. Younossi Z, Anstee QM, Marietti M, et al. Global burden of NAFLD and NASH: trends, predictions, risk factors and prevention. Nature reviews Gastroenterology \& hepatology. 2018;15(1):11-20.

10. Parnell JA, Raman M, Rioux KP, et al. The potential role of prebiotic fibre for treatment and management of non-alcoholic fatty liver disease and associated obesity and insulin resistance. Liver international : official journal of the International Association for the Study of the Liver. 2012;32(5):701-11.

11. Kim TJ, Sinn DH, Min YW, et al. A cohort study on Helicobacter pylori infection associated with nonalcoholic fatty liver disease. Journal of gastroenterology. 2017;52(11):1201-10.

12. Cai $\mathrm{O}$, Huang $\mathrm{Z}$, Li M, et al. Association between Helicobacter pylori Infection and Nonalcoholic Fatty Liver Disease: A Single-Center Clinical Study. Gastroenterology research and practice. 2018;2018:8040262.

13. $\mathrm{Li} \mathrm{M}$, Shen $\mathrm{Z}, \mathrm{Li} \mathrm{YM}$. Potential role of Helicobacter pylori infection in nonalcoholic fatty liver disease. World J Gastroenterol. 2013;19(41):7024-31.

14. Fan JG, Jia JD, Li YM, et al. Guidelines for the diagnosis and management of nonalcoholic fatty liver disease: update 2010: (published in Chinese on Chinese Journal of Hepatology 2010; 18:163-166). Journal of digestive diseases. 2011;12(1):38-44.

15. Chalasani N, Younossi Z, Lavine JE, et al. The diagnosis and management of non-alcoholic fatty liver disease: practice guideline by the American Gastroenterological Association, American Association for the Study of Liver Diseases, and American College of Gastroenterology. Gastroenterology. 2012;142(7):1592-609.

16. Ferraioli G, Soares Monteiro LB. Ultrasound-based techniques for the diagnosis of liver steatosis. World J Gastroenterol. 2019;25(40):6053-62.

17. Mantovani A, Turino T, Altomari A, et al. Association between Helicobacter pylori infection and risk of nonalcoholic fatty liver disease: An updated meta-analysis. Metabolism: clinical and experimental. 2019;96:56-65.

18. Okushin $\mathrm{K}$, Takahashi $\mathrm{Y}$, Yamamichi $\mathrm{N}$, et al. Helicobacter pylori infection is not associated with fatty liver disease including non-alcoholic fatty liver disease: a large-scale cross-sectional study in Japan. BMC gastroenterology. 2015;15:25.

19. Fan N, Peng L, Xia Z, et al. Helicobacter pylori Infection Is Not Associated with Non-alcoholic Fatty Liver Disease: A Cross-Sectional Study in China. Frontiers in microbiology. 2018;9:73.

20. Ballestri S, Nascimbeni F, Baldelli E et al. NAFLD as a Sexual Dimorphic Disease: Role of Gender and Reproductive Status in the Development and Progression of Nonalcoholic Fatty Liver Disease and Inherent Cardiovascular Risk. Advances in therapy. 2017;34(6):1291-326.

21. Hofbauer LC, Hamann C, Ebeling PR. Approach to the patient with secondary osteoporosis. European Society of Endocrinology. 2010;162(6):1009-20.

22. Lim SH, Kim N, Kwon JW, et al. Positive Association Between Helicobacter pylori Infection and Metabolic Syndrome in a Korean Population: A 
Multicenter Nationwide Study. Digestive diseases and sciences. 2019;64(8): 2219-30.

23. Refaeli R, Chodick G, Haj S, et al. Relationships of H. pylori infection and its related gastroduodenal morbidity with metabolic syndrome: a large cross-sectional study. Scientific reports. 2018;8(1):4088.

24. Fabbrini E, Magkos F. Hepatic Steatosis as a Marker of Metabolic Dysfunction. Nutrients. 2015;7(6):4995-5019.

25. Gunji T, Matsuhashi N, Sato $\mathrm{H}$, et al. Helicobacter pylori infection is significantly associated with metabolic syndrome in the Japanese population. The American journal of gastroenterology. 2008;103(12):3005-10.

26. Kim TJ, Lee H, Kang M, et al. Helicobacter pylori is associated with dyslipidemia but not with other risk factors of cardiovascular disease. Scientific reports. 2016;6:38015.

27. Satoh H, Saijo Y, Yoshioka E, et al. Helicobacter Pylori infection is a significant risk for modified lipid profile in Japanese male subjects. Journal of atherosclerosis and thrombosis. 2010;17(10):1041-8.

28. Chen C, Zhang C, Wang X, et al. Helicobacter pylori infection may increase the severity of nonalcoholic fatty liver disease via promoting liver function damage, glycometabolism, lipid metabolism, inflammatory reaction and metabolic syndrome. European journal of gastroenterology \& hepatology. 2020;32(7):857-66

29. Polyzos SA, Kountouras J, Zavos C, et al. The association between Helicobacter pylori infection and insulin resistance: a systematic review. Helicobacter. 2011:16(2):79-88.

30. Okushin K, Tsutsumi T, Ikeuchi K, et al. Helicobacter pylori infection and liver diseases: Epidemiology and insights into pathogenesis. World J Gastroenterol. 2018;24(32):3617-25

31. $\mathrm{Li} \mathrm{JZ,} \mathrm{Li} \mathrm{JY,} \mathrm{Wu} \mathrm{TF,} \mathrm{et} \mathrm{al.} \mathrm{Helicobacter} \mathrm{pylori} \mathrm{Infection} \mathrm{Is} \mathrm{Associated} \mathrm{with} \mathrm{Type}$ 2 Diabetes, Not Type 1 Diabetes: An Updated Meta-Analysis. Gastroenterology research and practice. 2017;2017:5715403.

32. Nilsson $\mathrm{HO}$, Taneera J, Castedal M, et al. Identification of Helicobacter pylori and other Helicobacter species by PCR, hybridization, and partial DNA sequencing in human liver samples from patients with primary sclerosing cholangitis or primary biliary cirrhosis. Journal of clinical microbiology. 2000;38(3):1072-6.

33. Castaño-Rodríguez N, Mitchell HM, Kaakoush NO. NAFLD, Helicobacter species and the intestinal microbiome. Best practice \& research Clinical gastroenterology. 2017;31(6):657-68.

34. Cheng DD, He C, Ai HH, et al. The Possible Role of Helicobacter pylori Infection in Non-alcoholic Fatty Liver Disease. Frontiers in microbiology. 2017;8:743.

35. Miele L, Valenza V, La Torre G, et al. Increased intestinal permeability and tight junction alterations in nonalcoholic fatty liver disease. Hepatology (Baltimore, Md). 2009;49(6):1877-87.

36. McColl KE. Clinical practice. Helicobacter pylori infection. The New England journal of medicine. 2010;362(17):1597-604.

37. Maharshi V, Gupta P, Kumar VL, et al. Effect of Helicobacter pylorieradication therapy on hepatic steatosis in patients with non-alcoholic fatty liver disease: a randomized-controlled pilot study. Gastroenterology report. 2020;8(2):104-10

38. Polyzos SA, Nikolopoulos P, Stogianni A, et al. Effect of Helicobacter pylori eradication on hepatic steatosis, NAFLD fibrosis score and HSENSI in patients with nonalcoholic steatohepatitis: a MR imaging-based pilot open-label study. Arquivos de gastroenterologia. 2014;51(3):261-8. 